\title{
Approximation of Conformal Mapping via the Szegő Kernel Method
}

\author{
Igor E. Pritsker \\ (Communicated by Vladimir V. Andrievskii) \\ Dedicated to the memory of Professor D. Gaier
}

\begin{abstract}
We study the uniform approximation of the canonical conformal mapping, for a Jordan domain onto the unit disk, by polynomials generated from the partial sums of the Szegö kernel expansion. These polynomials converge to the conformal mapping uniformly on the closure of any Smirnov domain. We prove estimates for the rate of such convergence on domains with piecewise analytic boundaries, expressed through the smallest exterior angle at the boundary. Furthermore, we show that the rate of approximation on compact subsets inside the domain is essentially the square of that on the closure. Two standard applications to the rate of decay for the contour orthogonal polynomials inside the domain, and to the rate of locally uniform convergence of Fourier series are also given.
\end{abstract}

Keywords. Conformal mapping, Szegő kernel, Fourier series, orthogonal polynomials.

2000 MSC. Primary 30C40, 30E10; Secondary 41A10, 30C30.

\section{Convergence of the Szegö kernel expansion and approximation of conformal maps}

Let $G$ be a Jordan domain in the complex plane. There are two well known kernel methods used for approximation of the canonical conformal mappings of $G$ onto a disk. The Bergman kernel method is associated with the $L_{2}$ spaces and orthogonal polynomials with respect to the area measure, while the Szegö kernel method is based on the inner product and orthogonal polynomials with respect to the arclength measure on the boundary of $G$ (see Gaier [7], Smirnov and Lebe$\operatorname{dev}[[1.9])$. The approximations related to the area orthogonality approach were

Received May 6, 2003.

This material is based upon work supported by the National Science Foundation under Grant No. 9996410, and by the National Security Agency under Grant No. MDA904-03-1-0081.

ISSN 1617-9447/\$2.50 (C) 2003 Heldermann Verlag 
first introduced by Bieberbach [5] through an extremal problem for polynomials. The first result about the uniform convergence of the Bieberbach polynomials was proved by Keldysh [14], for domains with sufficiently smooth boundaries. The uniform convergence of the Bieberbach polynomials has been extensively studied since then (see Gaier [ 8$]$ and references therein). A selection of further results on this subject is in the papers of Andrievskii [1], Gaier [9]-[1T], Andrievskii and Gaier [3], and Andrievskii and Pritsker [4]. In contrast however, the method based on the Szegö kernel did not receive any comprehensive attention. The goal of this paper is to show that the Szegö kernel method is also very useful for the uniform approximation of conformal mappings.

Suppose that $G$ has rectifiable boundary $L$ of length $l$. We consider the Smirnov spaces $E_{p}(G), 1 \leq p<\infty$, of analytic functions in $G$, whose boundary values satisfy

$$
\|f\|_{p}=\left(\frac{1}{l} \int_{L}|f(z)|^{p}|d z|\right)^{1 / p}<\infty
$$

(see Duren [6], Smirnov and Lebedev [1[9]). Our interest is focused on the Hilbert space $E_{2}(G)$, equipped with the inner product

$$
(f, g):=\frac{1}{l} \int_{L} f(z) \overline{g(z)}|d z|, \quad f, g \in E_{2}(G) .
$$

Polynomials are dense in $E_{p}(G), 1 \leq p<\infty$, if and only if $G$ is a Smirnov domain [6]. Keldysh and Lavrentiev [15] characterized Smirnov domains by the property that, for a conformal mapping $\psi$ of the unit disk $\mathbb{D}$ onto $G, \log |\psi|$ is represented by the Poisson integral of its boundary values. Although no complete geometric description of Smirnov domains is known, this class is sufficiently wide. In particular, it contains (in the decreasing order of generality) all Ahlfors-regular domains, Lavrentiev (cord-arc) domains, Lipschitz domains, domains with bounded boundary rotation (Radon domains), piecewise smooth and smooth domains (cf. Pommerenke [17, Chap. 7]). Applying Gram-Schmidt orthonormalization to monomials $\left\{z^{n}\right\}_{n=0}^{\infty}$ in the Smirnov domain $G$, we obtain a complete orthonormal system of polynomials $\left\{p_{n}(z)\right\}_{n=0}^{\infty}$ in $E_{2}(G)$ (see Szegö [2]]). Next, we introduce the Szegö kernel

$$
K(z, \zeta)=\sum_{k=0}^{\infty} \overline{p_{k}(\zeta)} p_{k}(z), \quad z, \zeta \in G,
$$

where convergence of this bilinear series is uniform in $z$ and $\zeta$ on compact subsets in $G$ [27], [19] , [7]. The importance of the Szegö kernel lies in its reproducing property

$$
f(\zeta)=\frac{1}{l} \int_{L} f(z) \overline{K(z, \zeta)}|d z|, \quad \zeta \in G
$$


which holds for any $f \in E_{2}(G)$. Equivalently, every $f \in E_{2}(G)$ can be represented by its Fourier series

$$
f(\zeta)=\sum_{k=0}^{\infty} a_{k} p_{k}(\zeta)=\sum_{k=0}^{\infty}\left(\frac{1}{l} \int_{L} f(z) \overline{p_{k}(z)}|d z|\right) p_{k}(\zeta)
$$

convergent in the $E_{2}(G)$ norm and, consequently, locally uniformly convergent in $G$ (see [19, Chapter 4]).

It is well known that the Szegö kernel is closely connected with the canonical conformal mapping $\varphi$ of $G$ onto the unit disk $\mathbb{D}$ :

$$
K(z, \zeta)=\frac{l}{2 \pi} \sqrt{\varphi^{\prime}(z) \varphi^{\prime}(\zeta)}
$$

where $\varphi(\zeta)=0$ and $\varphi^{\prime}(\zeta)>0$ (cf. [19] and [21]). Hence we have that

$$
\varphi^{\prime}(\zeta)=\frac{2 \pi}{l} K(\zeta, \zeta)=\frac{2 \pi}{l} \sum_{k=0}^{\infty}\left|p_{k}(\zeta)\right|^{2},
$$

by $(1.1)$ and (1.3). It follows that

$$
\varphi^{\prime}(z)=\frac{2 \pi}{l} \frac{(K(z, \zeta))^{2}}{K(\zeta, \zeta)}=\frac{2 \pi}{l} \frac{\left(\sum_{k=0}^{\infty} \overline{p_{k}(\zeta)} p_{k}(z)\right)^{2}}{\sum_{k=0}^{\infty}\left|p_{k}(\zeta)\right|^{2}}, \quad z \in G,
$$

where $\zeta \in G$ is regarded as a fixed point. We now introduce the following sequence of approximating polynomials.

$$
J_{2 n+1}(z)=\frac{2 \pi}{l} \frac{\int_{\zeta}^{z}\left(\sum_{k=0}^{n} \overline{p_{k}(\zeta)} p_{k}(t)\right)^{2} d t}{\sum_{k=0}^{n}\left|p_{k}(\zeta)\right|^{2}}, \quad n \in \mathbb{N} .
$$

Note that the degree of $J_{2 n+1}(z)$ is $2 n+1$. The sequence $\left\{J_{2 n+1}\right\}_{n=0}^{\infty}$ converges to $\varphi$ uniformly on compact subsets of $G$, which is inherited from the partial sums of the Szegő kernel.

Similar approximating polynomials, but with a different normalization, were introduced via an extremal problem for any $E_{p}(\Gamma), 1 \leq p<\infty$, by Keldysh and Lavrentiev (see [1.3] and [1.5]). They developed the ideas of Julia [12], who earlier considered the same extremal problem for the conformal mapping. Further study of the convergence properties in $E_{2}(G)$ is due to Warschawski [22] (also see Gaier [7] for a survey). Convergence questions for general Fourier expansions in contour orthogonal polynomials were considered in Rosenbloom and Warschawski [18], Smirnov and Lebedev [19] and Suetin [20]. However, all of these investigations impose quite strict smoothness assumptions on the boundary of $G$. We prove the first uniform convergence results for domains with corners, 
and give explicit rates of approximation in terms of the geometric properties of $G$.

We start with a general estimate for the uniform (sup) norm of the approximation error on $\bar{G}$. A similar result was proved by Warschawski [22], but in a somewhat different form (see also [7, pp. 130-131]).

Theorem 1.1. Let $G$ be a Smirnov domain, with $\zeta \in G$ fixed. If $\varphi: G \rightarrow \mathbb{D}$ is a conformal mapping normalized by $\varphi(\zeta)=0$ and $\varphi^{\prime}(\zeta)>0$, then

$$
\left\|\varphi-J_{2 n+1}\right\|_{\infty} \leq 8 \pi\left\|K(\cdot, \zeta)-\sum_{k=0}^{n} \overline{p_{k}(\zeta)} p_{k}(\cdot)\right\|_{2} \rightarrow 0 \quad \text { as } n \rightarrow \infty .
$$

Recall that $\sum_{k=0}^{n} \overline{p_{k}(\zeta)} p_{k}(z)$ is the Fourier sum for $K(z, \zeta)$, i.e., it is the best $E_{2}(G)$ approximation from the subspace of polynomials of degree $n$. Hence we can give an upper estimate for the rate of convergence by appropriately choosing a sequence of approximating polynomials for $K(z, \zeta)$. The rate of convergence necessarily depends on the geometric properties of the domain $G$. We consider a class of domains with piecewise analytic boundaries, which is important in applications. An analytic arc is defined as the image of a segment under a mapping that is conformal in an open neighborhood of the segment. Thus a domain has piecewise analytic boundary if it is bounded by a Jordan curve consisting of a finite number of analytic arcs.

Theorem 1.2. Let $\partial G$ be piecewise analytic, with the smallest exterior angle $\lambda \pi, 0<\lambda<2$, at the junction points of the analytic arcs. If $\zeta \in F$, where $F \subset G$ is compact, then

$$
\left\|K(\cdot, \zeta)-\sum_{k=0}^{n} \overline{p_{k}(\zeta)} p_{k}(\cdot)\right\|_{2} \leq C_{1}(G, F) n^{-\lambda /(4-2 \lambda)}, \quad n \in \mathbb{N} .
$$

Here, the constant $C_{1}(G, F)>0$ depends only on $G$ and $F$.

Combining Theorems 1.1 and 1.2, we obtain the main result on the uniform approximation of conformal mappings.

Theorem 1.3. Let $\partial G$ be piecewise analytic, with the smallest exterior angle $\lambda \pi, 0<\lambda<2$, at the junction points of the analytic arcs. Suppose that $\zeta \in F$, where $F \subset G$ is compact. For the conformal mapping $\varphi: G \rightarrow \mathbb{D}$, normalized by $\varphi(\zeta)=0$ and $\varphi^{\prime}(\zeta)>0$, we have

$$
\left\|\varphi-J_{2 n+1}\right\|_{\infty} \leq C_{2}(G, F) n^{-\lambda /(4-2 \lambda)}, \quad n \in \mathbb{N},
$$

where the constant $C_{2}(G, F)>0$ depends only on $G$ and $F$.

It is interesting to compare the convergence properties of our sequence $\left\{J_{2 n+1}\right\}_{n=0}^{\infty}$ with those of the Bieberbach polynomials $\left\{B_{n}\right\}_{n=0}^{\infty}$. Gaier [10] proved for domains with piecewise analytic boundaries that

$$
\left\|\varphi-B_{n}\right\|_{\infty} \leq C_{3} \log n n^{-\lambda /(2-\lambda)}, \quad n \geq 2 .
$$


Later, Andrievskii and Gaier [3] replaced $\log n$ by $(\log n)^{1 / 2}$, and relaxed the imposed geometric condition to piecewise quasianalytic boundary. Their estimate for the rate of uniform convergence of the Bieberbach polynomials remains the most precise known. Although (1.8) gives a slower rate of convergence, the polynomials $J_{2 n+1}$ have some advantages over the Bieberbach polynomials. They are free from the convergence anomalies exhibited by Keldysh's example [14], where only one singular point, at an otherwise very smooth boundary, destroys the uniform convergence of the Bieberbach polynomials. (One can find further information about this example of Keldysh in [4].) In fact, Smirnov domains include any imaginable domain arising in numerical applications, guaranteeing the uniform convergence of $\left\{J_{2 n+1}\right\}_{n=0}^{\infty}$ by Theorem 1.1. In addition, this sequence is easier to generate numerically, because the inner products defined by the boundary integrals are easier to compute than the area inner products in Gram-Schmidt orthonormalization.

We show that the rate of convergence for $J_{2 n+1}$ on compact subsets of $G$ is better than that on the whole domain, i.e., it is essentially squared compared to (1.8).

Theorem 1.4. If the conditions of Theorem 1.3 are satisfied, then

$$
\max _{z \in F}\left|\varphi(z)-J_{2 n+1}(z)\right| \leq C_{4}(G, F) n^{-\lambda /(2-\lambda)}, \quad n \in \mathbb{N},
$$

where $C_{4}(G, F)>0$ depends only on $G$ and $F$.

Gaier [II] posed the question of possible improvement in locally uniform convergence rates for the Bieberbach polynomials and other approximations for conformal maps. The above theorem provides a partial answer for his question in the case of the Szego" kernel method. Furthermore, it is possible to give similar improvements for the Bieberbach polynomials too, by following the ideas of this paper.

Clearly, it was not our goal to achieve the highest possible level of generality here. Thus Theorem 1.2 is true for domains with piecewise quasianalytic boundaries [3] (therefore, all other results are valid for these domains too). One only needs to fill in a number of technical details on the behaviour of $\left(\varphi^{\prime}\right)^{1 / 2}$ near corners, in our proof, to reach this conclusion. We are also able to prove Theorems $1.2-1.4$ for Lavrentiev (cord-arc) domains, with the rates of convergence of the order $n^{-\gamma}, \gamma>0$. Finally, we have analogues of these results in $E_{p}(G)$ for $p \neq 2$.

\section{Orthogonal polynomials and Fourier series}

We give two standard applications for approximation of the Szegö kernel here. Since polynomials $\left\{p_{n}\right\}_{n=0}^{\infty}$ form a complete orthonormal system, we can restate (1.7) in the following form.

$$
\left(\sum_{k=n+1}^{\infty}\left|p_{k}(\zeta)\right|^{2}\right)^{1 / 2} \leq C_{1}(G, F) n^{-\lambda /(4-2 \lambda)}, \quad n \in \mathbb{N},
$$


by (1.1). It is well known that $p_{n}$ converge to zero on compact subsets of $G$ as $n \rightarrow \infty$ (cf. [2I, Chapter XVI]). Equation (2.1) immediately gives an estimate for the rate of decay of $p_{n}$ inside $G$.

Theorem 2.1. Let $\partial G$ be piecewise analytic, with the smallest exterior angle $\lambda \pi$, $0<\lambda<2$, at the junction points of the analytic arcs. If $\zeta \in F$, where $F \subset G$ is compact, then

$$
\left|p_{n}(\zeta)\right| \leq C_{1}(G, F) n^{-\lambda /(4-2 \lambda)}, \quad n \in \mathbb{N}
$$

The second application is related to the rates of convergence of the Fourier series (1.2) for $f \in E_{2}(G)$ on compact subsets of $G$. Observe that

$$
\|f\|_{2}=\left(\sum_{k=0}^{\infty}\left|a_{k}\right|^{2}\right)^{1 / 2} .
$$

Hence we have from (1.2) and Cauchy-Schwarz inequality that

$$
\begin{aligned}
\left|f(\zeta)-\sum_{k=0}^{n} a_{k} p_{k}(\zeta)\right| & =\left|\sum_{k=n+1}^{\infty} a_{k} p_{k}(\zeta)\right| \\
& \leq\left(\sum_{k=n+1}^{\infty}\left|a_{k}\right|^{2}\right)^{1 / 2}\left(\sum_{k=n+1}^{\infty}\left|p_{k}(\zeta)\right|^{2}\right)^{1 / 2} \\
& =\left\|f-\sum_{k=0}^{n} a_{k} p_{k}\right\|_{2}\left(\sum_{k=n+1}^{\infty}\left|p_{k}(\zeta)\right|^{2}\right)^{1 / 2} \\
& \leq\|f\|_{2}\left(\sum_{k=n+1}^{\infty}\left|p_{k}(\zeta)\right|^{2}\right)^{1 / 2} .
\end{aligned}
$$

We thus obtain the following result from (2.1).

Theorem 2.2. Let $\partial G$ be piecewise analytic, with the smallest exterior angle $\lambda \pi$, $0<\lambda<2$, at the junction points of the analytic arcs. Suppose that $f \in E_{2}(G)$ has the Fourier expansion (1.2). If $\zeta \in F$, where $F \subset G$ is compact, then

$$
\left|f(\zeta)-\sum_{k=0}^{n} a_{k} p_{k}(\zeta)\right| \leq C_{1}(G, F) n^{-\lambda /(4-2 \lambda)}\left\|f-\sum_{k=0}^{n} a_{k} p_{k}\right\|_{2}, \quad n \in \mathbb{N} .
$$

Results of this kind for domains with smooth boundaries were previously proved by Szegö [21], Rosenbloom and Warschawski [18], Smirnov and Lebedev [1.9], Suetin [20], and others. 


\section{Proofs}

Proof of Theorem 1.1. Let $\psi:=\varphi^{-1}$. We have

$$
\begin{aligned}
\left|\varphi(z)-J_{2 n+1}(z)\right| & =\left|\int_{\zeta}^{z}\left(\varphi^{\prime}(t)-J_{2 n+1}^{\prime}(t)\right) d t\right| \\
& =\left|\int_{0}^{\varphi(z)}\left(\varphi^{\prime}(\psi(u))-J_{2 n+1}^{\prime}(\psi(u))\right) \psi^{\prime}(u) d u\right| \\
& \leq \int_{0}^{\varphi(z)}\left|\left(\varphi^{\prime}(\psi(u))-J_{2 n+1}^{\prime}(\psi(u))\right) \psi^{\prime}(u)\right||d u|,
\end{aligned}
$$

where the integration is carried over the segment connecting 0 and $\varphi(z)$ in $\mathbb{D}$. Note that the function $\left(\varphi^{\prime}(\psi(u))-J_{2 n+1}^{\prime}(\psi(u))\right) \psi^{\prime}(u)=1-J_{2 n+1}^{\prime}(\psi(u)) \psi^{\prime}(u)$ belongs to the Hardy class $H^{1}(\mathbb{D})$, because $L$ is rectifiable. Hence we obtain by Fejér-Riesz inequality (cf. [6, Theorem 3.13]) that

$$
\begin{aligned}
\left|\varphi(z)-J_{2 n+1}(z)\right| & \leq \frac{1}{2} \int_{|u|=1} \mid\left(\varphi^{\prime}(\psi(u))-J_{2 n+1}^{\prime}(\psi(u)) \psi^{\prime}(u)|| d u \mid\right. \\
& =\frac{1}{2} \int_{L}\left|\varphi^{\prime}(t)-J_{2 n+1}^{\prime}(t)\right||d t| .
\end{aligned}
$$

Define

$$
Q_{n}(z):=\left(\frac{l}{2 \pi} \sum_{k=0}^{n}\left|p_{k}(\zeta)\right|^{2}\right)^{-1 / 2} \sum_{k=0}^{n} \overline{p_{k}(\zeta)} p_{k}(z),
$$

so that $J_{2 n+1}^{\prime}(z)=Q_{n}{ }^{2}(z)$. We continue with this notation, by using the CauchySchwarz and Minkowski inequalities.

$$
\begin{aligned}
\left|\varphi(z)-J_{2 n+1}(z)\right| & \leq \frac{1}{2} \int_{L}\left|\left(\sqrt{\varphi^{\prime}(t)}\right)^{2}-Q_{n}{ }^{2}(t)\right||d t| \\
& =\frac{1}{2} \int_{L}\left|\sqrt{\varphi^{\prime}(t)}-Q_{n}(t)\right|\left|\sqrt{\varphi^{\prime}(t)}+Q_{n}(t)\right||d t| \\
& \leq \frac{l}{2}\left\|\sqrt{\varphi^{\prime}}-Q_{n}\right\|_{2}\left\|\sqrt{\varphi^{\prime}}+Q_{n}\right\|_{2} \\
& \leq \frac{l}{2}\left\|\sqrt{\varphi^{\prime}}-Q_{n}\right\|_{2}\left(\left\|\sqrt{\varphi^{\prime}}\right\|_{2}+\left\|Q_{n}\right\|_{2}\right) .
\end{aligned}
$$

Observe that

$$
\left\|\sqrt{\varphi^{\prime}}\right\|_{2}=\left(\frac{1}{l} \int_{L}\left|\varphi^{\prime}(z)\right||d z|\right)^{1 / 2}=\left(\frac{1}{l} \int_{|w|=1}|d w|\right)^{1 / 2}=\sqrt{\frac{2 \pi}{l}}
$$

and that

$$
\left\|Q_{n}\right\|_{2}=\left(\frac{l}{2 \pi} \sum_{k=0}^{n}\left|p_{k}(\zeta)\right|^{2}\right)^{-1 / 2}\left(\sum_{k=0}^{n}\left|p_{k}(\zeta)\right|^{2}\right)^{1 / 2}=\sqrt{\frac{2 \pi}{l}}
$$


by orthonormality of the polynomials $p_{k}$. Thus

$$
\left\|\varphi-J_{2 n+1}\right\|_{\infty} \leq \sqrt{2 \pi l}\left\|\sqrt{\varphi^{\prime}}-Q_{n}\right\|_{2} .
$$

We now estimate the norm on the right of the above inequality. Recall that

$$
\sqrt{\varphi^{\prime}(z)}=\sqrt{\frac{2 \pi}{l}} \frac{K(z, \zeta)}{\sqrt{K(\zeta, \zeta)}},
$$

by (1.5). Therefore,

$$
\begin{aligned}
& \sqrt{\varphi^{\prime}(z)}-Q_{n}(z)=\sqrt{\frac{2 \pi}{l}}\left(\frac{K(z, \zeta)}{\sqrt{K(\zeta, \zeta)}}-\left(\sum_{k=0}^{n}\left|p_{k}(\zeta)\right|^{2}\right)^{-1 / 2} \sum_{k=0}^{n} \overline{p_{k}(\zeta)} p_{k}(z)\right) \\
& =\sqrt{\frac{2 \pi}{l K(\zeta, \zeta)}}\left(K(z, \zeta)-\sum_{k=0}^{n} \overline{p_{k}(\zeta)} p_{k}(z)\right) \\
& +\sqrt{\frac{2 \pi}{l K(\zeta, \zeta)}}\left(1-\left(\frac{K(\zeta, \zeta)}{\sum_{k=0}^{n}\left|p_{k}(\zeta)\right|^{2}}\right)^{1 / 2}\right) \sum_{k=0}^{n} \overline{p_{k}(\zeta)} p_{k}(z) .
\end{aligned}
$$

It follows that

$$
\begin{aligned}
& \left\|\sqrt{\varphi^{\prime}}-Q_{n}\right\|_{2} \leq \sqrt{\frac{2 \pi}{l K(\zeta, \zeta)}}\left\|K(\cdot, \zeta)-\sum_{k=0}^{n} \overline{p_{k}(\zeta)} p_{k}(\cdot)\right\|_{2} \\
& +\sqrt{\frac{2 \pi}{l K(\zeta, \zeta)}}\left(\left(\frac{K(\zeta, \zeta)}{\sum_{k=0}^{n}\left|p_{k}(\zeta)\right|^{2}}\right)^{1 / 2}-1\right)\left(\sum_{k=0}^{n}\left|p_{k}(\zeta)\right|^{2}\right)^{1 / 2}
\end{aligned}
$$

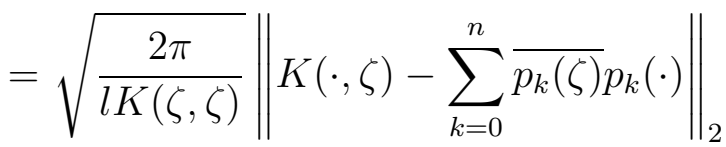

$$
\begin{aligned}
& +\sqrt{\frac{2 \pi}{l K(\zeta, \zeta)}}\left((K(\zeta, \zeta))^{1 / 2}-\left(\sum_{k=0}^{n}\left|p_{k}(\zeta)\right|^{2}\right)^{1 / 2}\right) .
\end{aligned}
$$

Since

$$
\begin{aligned}
(K(\zeta, \zeta))^{1 / 2}-\left(\sum_{k=0}^{n}\left|p_{k}(\zeta)\right|^{2}\right)^{1 / 2} & =\|K(\cdot, \zeta)\|_{2}-\left\|\sum_{k=0}^{n} \overline{p_{k}(\zeta)} p_{k}(\cdot)\right\|_{2} \\
& \leq\left\|K(\cdot, \zeta)-\sum_{k=0}^{n} \overline{p_{k}(\zeta)} p_{k}(\cdot)\right\|_{2}
\end{aligned}
$$


we obtain that

$$
\left\|\sqrt{\varphi^{\prime}}-Q_{n}\right\|_{2} \leq 2 \sqrt{\frac{2 \pi}{l K(\zeta, \zeta)}}\left\|K(\cdot, \zeta)-\sum_{k=0}^{n} \overline{p_{k}(\zeta)} p_{k}(\cdot)\right\|_{2}
$$

Equation (3.1) now gives that

$$
\left\|\varphi-J_{2 n+1}\right\|_{\infty} \leq \frac{4 \pi}{\sqrt{K(\zeta, \zeta)}}\left\|K(\cdot, \zeta)-\sum_{k=0}^{n} \overline{p_{k}(\zeta)} p_{k}(\cdot)\right\|_{2}
$$

Recall that

$$
K(\zeta, \zeta)=\frac{l}{2 \pi} \varphi^{\prime}(\zeta)
$$

by (1.4). Using [17, Corollary 1.4, p. 9], we have

$$
\left|\varphi^{\prime}(\zeta)\right| \geq \frac{1}{4} \frac{1-|\varphi(\zeta)|^{2}}{\operatorname{dist}(\zeta, L)}=\frac{1}{4 \operatorname{dist}(\zeta, L)},
$$

where $\operatorname{dist}(\zeta, L)$ is the distance from $\zeta$ to $L$. Clearly, $l \geq 2 \pi \operatorname{dist}(\zeta, L)$, so that

$$
K(\zeta, \zeta)=\frac{l \varphi^{\prime}(\zeta)}{2 \pi} \geq \varphi^{\prime}(\zeta) \operatorname{dist}(\zeta, L) \geq \frac{1}{4}
$$

Combining this with (3.2), we obtain (1.6).

Proof of Theorem 1.2. We start by recalling that the partial sum

$$
\sum_{k=0}^{n} \overline{p_{k}(\zeta)} p_{k}(z)
$$

for $K(z, \zeta)$ is its best approximation in $E_{2}(G)$ (with $\zeta \in G$ fixed) among all polynomials of degree at most $n$. Thus we construct a sequence of polynomials with good approximative properties, which gives the desired upper bound (1.7). It is clear from (1.3) that approximation of $K(z, \zeta)$ is equivalent to approximation of $\left(\varphi^{\prime}(z)\right)^{1 / 2}$. We use a method resembling that of Andrievskii and Gaier [3]. We first continue the mapping $\varphi$ conformally beyond the boundary $L$, by using reflections across the analytic $\operatorname{arcs} L_{i}, L=\bigcup_{i=1}^{m} L_{i}$. Suppose that $\tau_{i}$ is a mapping such that $L_{i}=\tau_{i}([0,1])$, which is conformal in an open neighborhood of $[0,1]$. Then we can find a symmetric lens shaped domain $S_{i}$, bounded by two circular arcs subtended by $[0,1]$, whose closure is contained in this open neighborhood of $[0,1]$. Defining

$$
\tilde{G}:=G \cup\left(\bigcup_{i=1}^{m} \tau_{i}\left(S_{i}\right)\right),
$$

we extend $\varphi$ into $\tilde{G}$ as follows.

$$
\varphi(z):=\frac{1}{\overline{\varphi\left[\tau_{i}\left(\overline{\tau_{i}^{-1}(z)}\right)\right]}}, \quad z \in \tau_{i}\left(S_{i}\right) \backslash \bar{G}
$$


where $i=1, \ldots, m$. The boundary $\partial \tilde{G}$ consists of $m$ analytic arcs $\Gamma_{i}$ that share endpoints with the $\operatorname{arcs} L_{i}$ of $\partial G$.

$$
\partial \tilde{G} \cap \partial G=\left\{z_{i}\right\}_{i=1}^{m},
$$

which are clearly the corner points of $\partial G$. Since each $\tau_{i}, i=1, \ldots, m$, is conformal and has bounded derivative (together with its inverse) on $S_{i}$, we obtain the inequalities

$$
\operatorname{dist}(z, \partial G) \geq c_{1} \min _{1 \leq i \leq m}\left|z-z_{i}\right|, \quad z \in \partial \tilde{G},
$$

where $\operatorname{dist}(z, \partial G)$ is the distance from $z$ to $\partial G$, and

$$
|\gamma| \leq c_{2}|z-t|, \quad z, t \in \partial \tilde{G}
$$

where $|\gamma|$ is the length of the shorter $\operatorname{arc} \gamma \subset \partial \tilde{G}$, connecting $z$ and $t$. We denote various positive constants by $c_{1}, c_{2}$, etc.

Let $\Gamma_{j}$ be an arc of $\partial \tilde{G}$, with the endpoints $z_{j}$ and $z_{j+1}$, and let $\zeta_{j} \in \Gamma_{j}$ be a fixed point, $j=1, \ldots, m$. Note that $\zeta_{j}$ divides $\Gamma_{j}$ into $\Gamma_{j}^{1}$ and $\Gamma_{j}^{2}$, so that

$$
\partial \tilde{G}=\bigcup_{j=1}^{m} \bigcup_{i=1}^{2} \Gamma_{j}^{i}
$$

We obtain from Cauchy's integral formula for the continuation of $\left(\varphi^{\prime}\right)^{1 / 2}$ into $\tilde{G}$ that

$$
\sqrt{\varphi^{\prime}(z)}=\frac{1}{2 \pi i} \int_{\partial \tilde{G}} \frac{\sqrt{\varphi^{\prime}(t)}}{t-z} d t=\frac{1}{2 \pi i} \sum_{j=1}^{m} \sum_{i=1}^{2} \int_{\Gamma_{j}^{i}} \frac{\sqrt{\varphi^{\prime}(t)}}{t-z} d t, \quad z \in \tilde{G} .
$$

Hence the problem is reduced to approximation of functions of the form

$$
g(z):=\int_{\gamma} \frac{\sqrt{\varphi^{\prime}(t)}}{t-z} d t
$$

in $E_{2}(G)$ norm, where $\gamma$ is any of the arcs $\Gamma_{j}^{i}$, with $i=1,2$ and $j=1, \ldots, m$.

Let $\Omega:=\overline{\mathbb{C}} \backslash \bar{G}$. Consider the standard conformal mapping $\Phi: \Omega \rightarrow \Delta$, where $\Delta:=\{w:|w|>1\}$, normalized by $\Phi(\infty)=\infty$ and $\Phi^{\prime}(\infty)>0$. We define the level curves of $\Phi$ by

$$
L_{n}:=\left\{z:|\Phi(z)|=1+\frac{1}{n}\right\}, \quad n \in \mathbb{N} .
$$

Denote by $\gamma_{2}$ the part of $\gamma$ from its endpoint $\zeta_{j} \in \Gamma_{j}$ to the first point $\xi$ of intersection with $L_{n}$, so that $\gamma_{2} \subset\{z:|\Phi(z)|>1+1 / n\}$. Then $\gamma_{1}:=\gamma \backslash \gamma_{2}$ connects $\xi$ with the corner point $z_{j}$ of $L$. Write

$$
g(z):=\int_{\gamma_{1}} \frac{\sqrt{\varphi^{\prime}(t)}}{t-z} d t+\int_{\gamma_{2}} \frac{\sqrt{\varphi^{\prime}(t)}}{t-z} d t=: g_{1}(z)+g_{2}(z) .
$$


We show that $\left\|g_{1}\right\|_{2} \rightarrow 0$ sufficiently fast as $n \rightarrow \infty$, while $g_{2}$ is well approximated by polynomials of degree $n$. To estimate the norm of $g_{1}$, we need to know the behaviour of $\left(\varphi^{\prime}\right)^{1 / 2}$ near the corner point $z_{j} \in L$. This is conveniently found from the asymptotic expansion of Lehman [16]. Assume that $z_{j}=0$ and that $\lambda_{j} \pi, 0<\lambda_{j}<2$, is the exterior angle formed by $L$ at this point. Then we have in a neighborhood of $z_{j}=0$ that

$$
\varphi(z)-\varphi(0)=b z^{1 /\left(2-\lambda_{j}\right)}+\mathcal{O}\left(z^{1 /\left(2-\lambda_{j}\right)}\right) \quad \text { as } z \rightarrow 0
$$

where $b \neq 0$, and

$$
\varphi^{\prime}(z)=\frac{b}{2-\lambda_{j}} z^{1 /\left(2-\lambda_{j}\right)-1}+\mathcal{O}\left(z^{1 /\left(2-\lambda_{j}\right)-1}\right) \quad \text { as } z \rightarrow 0 .
$$

Hence there exists a constant $c_{3}>0$ such that

$$
\left|\sqrt{\varphi^{\prime}(z)}\right| \leq c_{3}|z|^{\alpha}, \quad z \in \tilde{G} \cup \partial \tilde{G}
$$

where we set

$$
\alpha:=\frac{1}{4-2 \lambda_{j}}-\frac{1}{2} .
$$

For the endpoints $\xi \in L_{n}$ and 0 of $\gamma_{1}$, we let

$$
d_{n}:=|\xi-0|=|\xi| .
$$

It follows from (3.4) that

$$
\left|\gamma_{1}\right| \leq c_{2} d_{n}
$$

We now estimate that

$$
\left\|g_{1}\right\|_{2}^{2}=\frac{1}{l} \int_{L}\left|\int_{\gamma_{1}} \frac{\sqrt{\varphi^{\prime}(t)}}{t-z} d t\right|^{2}|d z| \leq c_{4} \int_{L}\left(\int_{\gamma_{1}} \frac{|t|^{\alpha}}{|t-z|}|d t|\right)^{2}|d z|
$$

by (3.5) and (3.6). Note that if $z \in L$ satisfies $|z| \geq d_{n}$, then $|t-z| \sim|z|$ by (3.3). Consequently,

$$
\begin{aligned}
\int_{L \cap\left\{|z| \geq d_{n}\right\}}\left(\int_{\gamma_{1}} \frac{|t|^{\alpha}}{|t-z|}|d t|\right)^{2}|d z| & \leq c_{5} \int_{L \cap\left\{|z| \geq d_{n}\right\}}\left(\frac{d_{n}{ }^{\alpha+1}}{|z|}\right)^{2}|d z| \\
& \leq c_{6} d_{n}{ }^{2 \alpha+1} .
\end{aligned}
$$


On the other hand, if $z \in L$ satisfies $|z| \leq d_{n}$, then $|t-z| \sim|t|+|z|$ by (3.3), and using (3.4) we obtain that

$$
\begin{aligned}
& \int_{L \cap\left\{|z| \leq d_{n}\right\}}\left(\int_{\gamma_{1}} \frac{|t|^{\alpha}}{|t-z|}|d t|\right)^{2}|d z| \\
& \leq c_{7} \int_{0}^{c_{8} d_{n}}\left(\int_{0}^{c_{9} d_{n}} \frac{s^{\alpha}}{s+r} d s\right)^{2} d r \\
& \leq c_{7} \int_{0}^{c_{8} d_{n}}\left(\int_{0}^{r} \frac{s^{\alpha}}{r} d s+\int_{r}^{c_{9} d_{n}} s^{\alpha-1} d s\right)^{2} d r \\
& \quad=c_{7} \int_{0}^{c_{8} d_{n}}\left(\frac{r^{\alpha}}{\alpha+1}+\frac{\left(c_{9} d_{n}\right)^{\alpha}-r^{\alpha}}{\alpha}\right)^{2} d r \\
& \leq c_{10} d_{n}^{2 \alpha+1}
\end{aligned}
$$

for $\alpha \neq 0$. If $\alpha=0$ then we estimate

$$
\begin{aligned}
\int_{L \cap\left\{|z| \leq d_{n}\right\}}\left(\int_{\gamma_{1}} \frac{|d t|}{|t-z|}\right)^{2}|d z| & =\int_{L \cap\left\{|z| \leq d_{n}\right\}}\left(\int_{\gamma_{1}} \frac{|t|^{1 / 2}|t|^{-1 / 2}}{|t-z|}|d t|\right)^{2}|d z| \\
& \leq c_{2} d_{n} \int_{L \cap\left\{|z| \leq d_{n}\right\}}\left(\int_{\gamma_{1}} \frac{|t|^{-1 / 2}}{|t-z|}|d t|\right)^{2}|d z| \\
& \leq c_{2} d_{n} c_{10} d_{n}{ }^{2(-1 / 2)+1}=c_{2} c_{10} d_{n}
\end{aligned}
$$

as above. Combining (3.7)-(3.9), we have that

$$
\left\|g_{1}\right\|_{2} \leq c_{11} d_{n}^{\alpha+1 / 2} \leq c_{11} d_{n}^{1 /(4-2 \lambda)},
$$

where $\lambda=\min _{1 \leq j \leq m} \lambda_{j}$.

The next step is the construction of approximating polynomials $P_{n}$ for $g_{2}$. This is accomplished by using Dzjadyk kernels (see, e.g. [2]) of the form

$$
K_{n}(t, z)=\sum_{i=0}^{n} a_{i}(t) z^{i}, \quad n \in \mathbb{N},
$$

which approximate the Cauchy kernel. It was proved in [3, Lemma 5] that a sequence of such kernels can be selected, so that for any fixed $m \in \mathbb{N}$, and for all $t \in \gamma$ with $|\Phi(t)| \geq 1+1 / n$, we have

$$
\left|\frac{1}{t-z}-K_{n}(t, z)\right| \leq c_{12} \frac{d_{n}{ }^{m}}{|t-z|^{m+1}}, \quad z \in L,
$$

for all sufficiently large $n \in \mathbb{N}$. In particular, (3.11) holds for $t \in \gamma_{2}$. Define the polynomials

$$
P_{n}(z):=\int_{\gamma_{2}} \sqrt{\varphi^{\prime}(t)} K_{n}(t, z) d t
$$


and estimate

$$
\begin{aligned}
\left\|g_{2}-P_{n}\right\|_{2}{ }^{2} & =\frac{1}{l} \int_{L}\left|\int_{\gamma_{2}}\left(\frac{1}{t-z}-K_{n}(t, z)\right) \sqrt{\varphi^{\prime}(t)} d t\right|^{2}|d z| \\
& \leq c_{13} d_{n}{ }^{2 m} \int_{L}\left(\int_{\gamma_{2}} \frac{|t|^{\alpha}}{|t-z|^{m+1}}|d t|\right)^{2}|d z|,
\end{aligned}
$$

by (3.11) and (3.6). Observe that $|t-z| \sim|t|+|z|$ for $t \in \gamma_{2}$. Therefore, we have for $m>\alpha$ that

$$
\begin{aligned}
& \int_{L}\left(\int_{\gamma_{2}} \frac{|t|^{\alpha}}{|t-z|^{m+1}}|d t|\right)^{2}|d z| \\
& \quad \leq c_{14} \int_{0}^{c_{15}}\left(\int_{c_{16} d_{n}}^{c_{17}} \frac{s^{\alpha}}{(s+r)^{m+1}} d s\right)^{2} d r \\
& \quad \leq c_{14} \int_{0}^{c_{16} d_{n}}\left(\int_{c_{16} d_{n}}^{c_{17}} s^{\alpha-m-1} d s\right)^{2} d r \\
& \quad+c_{14} \int_{c_{16} d_{n}}^{c_{15}}\left(r^{-m-1} \int_{c_{16} d_{n}}^{r} s^{\alpha} d s+\int_{r}^{c_{17}} s^{\alpha-m-1} d s\right)^{2} d r \\
& \quad \leq c_{18} d_{n}^{2(\alpha-m)+1}+c_{19} \int_{c_{16} d_{n}}^{c_{15}} r^{2(\alpha-m)} d r \\
& \leq c_{20} d_{n}^{2(\alpha-m)+1} .
\end{aligned}
$$

It follows that

$$
\left\|g_{2}-P_{n}\right\|_{2} \leq c_{21} d_{n}^{\alpha+1 / 2} \leq c_{21} d_{n}^{1 /(4-2 \lambda)} .
$$

Collecting (3.10), (3.12) and (3.5) together, we obtain

$$
\left\|g-P_{n}\right\|_{2} \leq\left\|g_{1}\right\|_{2}+\left\|g_{2}-P_{n}\right\|_{2} \leq c_{22} d_{n}{ }^{1 /(4-2 \lambda)} .
$$

Recall that $d_{n}=|\xi|$, where $\xi \in L_{n} \cap \gamma_{1}$. Applying the results of [16] to the conformal mapping $\Psi:=\Phi^{-1}$, we obtain

$$
z=\Psi(\Phi(z))-\Psi(\Phi(0))=a(\Phi(z)-\Phi(0))^{\lambda_{j}}+\mathcal{O}\left((\Phi(z)-\Phi(0))^{\lambda_{j}}\right) \quad \text { as } z \rightarrow 0,
$$

where $\lambda_{j} \pi$ is the exterior angle at $z_{j}=0$, and $a \neq 0$. Thus

$$
d_{n}=|\xi| \leq c_{23} \min _{z \in L_{n}}|z| \leq c_{24} n^{-\lambda_{j}} \leq c_{24} n^{-\lambda}, \quad n \in \mathbb{N}
$$

and

$$
\left\|g-P_{n}\right\|_{2} \leq c_{25} n^{-\lambda /(4-2 \lambda)}, \quad n \in \mathbb{N},
$$

by (3.13). Hence there exists a sequence of polynomials $Q_{n}$ such that

$$
\left\|\sqrt{\varphi^{\prime}}-Q_{n}\right\|_{2} \leq c_{26} n^{-\lambda /(4-2 \lambda)}, \quad n \in \mathbb{N} \text {. }
$$

Since

$$
K(z, \zeta)=\frac{l}{2 \pi} \sqrt{\varphi^{\prime}(z) \varphi^{\prime}(\zeta)}
$$


we obtain (1.7) from the previous estimate:

$$
\left\|K(\cdot, \zeta)-\frac{l}{2 \pi} \sqrt{\varphi^{\prime}(\zeta)} Q_{n}\right\|_{2} \leq \frac{c_{26} l}{2 \pi} \sqrt{\varphi^{\prime}(\zeta)} n^{-\lambda /(4-2 \lambda)}, \quad n \in \mathbb{N},
$$

where $\zeta \in G$ was fixed throughout this proof. We now lift this restriction and allow $\zeta$ to vary within a compact set $F \subset G$. Note that $\left|\varphi^{\prime}(\zeta)\right|$ is uniformly bounded on $F$, and so are the other constants in the above proof. Indeed, we only need to verify this for the constant $c_{3}$ of (3.6), arising from the first term in the expansion of $\varphi^{\prime}(z)$. One can obtain a conformal mapping $\tilde{\varphi}$ of $G$ onto $\mathbb{D}$ with $\tilde{\varphi}(\tilde{\zeta})=0$ and $\tilde{\varphi}^{\prime}(\tilde{\zeta})>0$, for any $\tilde{\zeta} \in F$, by composing $\varphi$ with a Möbius self-map of the unit disk, which is conformal in an open neighborhood of $\overline{\mathbb{D}}$. It follows that (3.6) holds for all such mappings $\tilde{\varphi}$ with a constant $c_{3}$ uniformly bounded for $\tilde{\zeta} \in F$.

Proof of Theorem 1.4. We proceed as in the proof of Theorem 1.1, denoting

$$
Q_{n}(z):=\left(\frac{l}{2 \pi} \sum_{k=0}^{n}\left|p_{k}(\zeta)\right|^{2}\right)^{-1 / 2} \sum_{k=0}^{n} \overline{p_{k}(\zeta)} p_{k}(z)
$$

so that $J_{2 n+1}^{\prime}(z)=Q_{n}{ }^{2}(z)$. It follows from (1.5) that

$$
\begin{aligned}
& \sqrt{\varphi^{\prime}(z)}-Q_{n}(z)=\sqrt{\frac{2 \pi}{l}}\left(\frac{K(z, \zeta)}{\sqrt{K(\zeta, \zeta)}}-\left(\sum_{k=0}^{n}\left|p_{k}(\zeta)\right|^{2}\right)^{-1 / 2} \sum_{k=0}^{n} \overline{p_{k}(\zeta)} p_{k}(z)\right) \\
& =\sqrt{\frac{2 \pi}{l K(\zeta, \zeta)}}\left(K(z, \zeta)-\sum_{k=0}^{n} \overline{p_{k}(\zeta)} p_{k}(z)\right) \\
& +\sqrt{\frac{2 \pi}{l K(\zeta, \zeta)}} \frac{\left(\sum_{k=0}^{n}\left|p_{k}(\zeta)\right|^{2}\right)^{1 / 2}-(K(\zeta, \zeta))^{1 / 2}}{\left(\sum_{k=0}^{n}\left|p_{k}(\zeta)\right|^{2}\right)^{1 / 2}} \sum_{k=0}^{n} \overline{p_{k}(\zeta)} p_{k}(z) .
\end{aligned}
$$

Recall that

$$
\lim _{n \rightarrow \infty} \sum_{k=0}^{n} \overline{p_{k}(\zeta)} p_{k}(z)=K(z, \zeta)
$$

where convergence is uniform for $z, \zeta \in F$, and that

$$
0<c_{1}<|K(z, \zeta)|<c_{2}<+\infty, \quad z, \zeta \in F
$$


Hence

$$
\begin{aligned}
\max _{z \in F}\left|\sqrt{\varphi^{\prime}(z)}-Q_{n}(z)\right| \leq & c_{3} \max _{z \in F}\left|K(z, \zeta)-\sum_{k=0}^{n} \overline{p_{k}(\zeta)} p_{k}(z)\right| \\
& +c_{4}\left((K(\zeta, \zeta))^{1 / 2}-\left(\sum_{k=0}^{n}\left|p_{k}(\zeta)\right|^{2}\right)^{1 / 2}\right) .
\end{aligned}
$$

The first term is estimated from (1.7)-(2.1) and the Cauchy-Schwarz inequality.

$$
\begin{aligned}
\max _{z, \zeta \in F}\left|K(z, \zeta)-\sum_{k=0}^{n} \overline{p_{k}(\zeta)} p_{k}(z)\right| & =\max _{z, \zeta \in F}\left|\sum_{k=n+1}^{\infty} \overline{p_{k}(\zeta)} p_{k}(z)\right| \\
& \leq\left(\sum_{k=n+1}^{\infty}\left|p_{k}(z)\right|^{2}\right)^{1 / 2}\left(\sum_{k=n+1}^{\infty}\left|p_{k}(\zeta)\right|^{2}\right)^{1 / 2} \\
& \leq\left(C_{1}(G, F)\right)^{2} n^{-\lambda /(2-\lambda)}, \quad n \in \mathbb{N} .
\end{aligned}
$$

Thus for the second term we also have

$$
\begin{aligned}
(K(\zeta, \zeta))^{1 / 2}-\left(\sum_{k=0}^{n}\left|p_{k}(\zeta)\right|^{2}\right)^{1 / 2} & \leq c_{5}\left(K(\zeta, \zeta)-\sum_{k=0}^{n}\left|p_{k}(\zeta)\right|^{2}\right) \\
& \leq c_{5}\left(C_{1}(G, F)\right)^{2} n^{-\lambda /(2-\lambda)}, \quad n \in \mathbb{N} .
\end{aligned}
$$

Combining these estimates, we obtain that

$$
\max _{z \in F}\left|\sqrt{\varphi^{\prime}(z)}-Q_{n}(z)\right| \leq c_{6} n^{-\lambda /(2-\lambda)}, \quad n \in \mathbb{N} .
$$

It immediately follows that

$$
\begin{aligned}
\max _{z \in F}\left|\varphi^{\prime}(z)-Q_{n}{ }^{2}(z)\right| & \leq \max _{z \in F}\left|\sqrt{\varphi^{\prime}(z)}+Q_{n}(z)\right| \max _{z \in F}\left|\sqrt{\varphi^{\prime}(z)}-Q_{n}(z)\right| \\
& \leq c_{7} n^{-\lambda /(2-\lambda)}, \quad n \in \mathbb{N},
\end{aligned}
$$

and that

$$
\max _{z \in F}\left|\varphi(z)-J_{2 n+1}(z)\right| \leq \max _{z \in F} \int_{\zeta}^{z}\left|\varphi^{\prime}(t)-Q_{n}{ }^{2}(t)\right||d t| \leq c_{8} n^{-\lambda /(2-\lambda)}, \quad n \in \mathbb{N},
$$

where all constants in this proof are independent of $z, \zeta \in F$ and $n \in \mathbb{N}$.

\section{References}

1. V. V. Andrievskii, Convergence of Bieberbach polynomials in domains with quasiconformal boundary, Ukrainian Math. J. 35 (1983), 233-236.

2. V. V. Andrievskii, V. I. Belyi, and V. K. Dzjadyk, Conformal Invariants in Constructive Theory of Functions of a Complex Variable, World Federation Publishers, Atlanta, 1995. 
3. V. V. Andrievskii and D. Gaier, Uniform convergence of Bieberbach polynomials in domains with piecewise quasianalytic boundary, Mitt. Math. Sem. Giessen 211 (1992), 49-60.

4. V. V. Andrievskii and I. E. Pritsker, Convergence of Bieberbach polynomials in domains with interior cusps, J. d'Analyse Math. 82 (2000), 315-332.

5. L. Bieberbach, Zur Theorie und Praxis der konformen Abbildung, Rend. Circ. Mat. Palermo 38 (1914), 98-112.

6. P. L. Duren, Theory of $H^{p}$ Spaces, Dover, New York, 2000.

7. D. Gaier, Konstruktive Methoden der konformen Abbildung, Springer-Verlag, Berlin, 1964.

8. __ Lectures on Complex Approximation, Birkhäuser, Boston, 1987.

9. Approx. 4 (1988), 289-305.

10. lytic boundary, Arch. Math. 58 (1992), 289-305.

11. __ Polynomial approximation of conformal maps, Constr. Approx. 14 (1998), 27-40.

12. G. Julia, Lecons sur la représentation conforme des aires simplement connexes, Paris, 1931.

13. M. V. Keldysh, On a class of extremal polynomials, Dokl. Akad. Nauk SSSR 4 (1936), 163-166 (in Russian).

14. 5 (1939), 391-400.

15. M. V. Keldysh and M. A. Lavrentiev, Sur la représentation conforme des domaines limités par des courbes rectifiables, Ann. Sci. École Norm. Sup. 54 (1937), 1-38.

16. R. S. Lehman, Development of the mapping function at an analytic corner, Pacific J. Math. 7 (1957), 1437-1449.

17. Ch. Pommerenke, Boundary Behaviour of Conformal Maps, Springer-Verlag, Berlin, 1992.

18. P. C. Rosenbloom and S. E. Warschawski, Approximation by polynomials, in Lectures on Functions of a Complex Variable, Ann Arbor, University of Michigan Press, 1955, 287-302.

19. V. I. Smirnov and N. A. Lebedev, Functions of a Complex Variable: Constructive Theory, MIT Press, Cambridge, 1968.

20. P. K. Suetin, Fundamental properties of polynomials orthogonal on a contour, Uspekhi Mat. Nauk 21 (1966), 41-88 (in Russian).

21. G. Szegő, Orthogonal Polynomials, Amer. Math. Soc., Providence, 1975.

22. S. E. Warschawski, Recent results in numerical methods of conformal mapping, in Proceedings of Symposia in Applied Mathematics, Vol. VI. Numerical Analysis, McGraw-Hill Book Company, Inc., New York, 1956, 219-250.

Igor E. Pritsker

E-MAIL: igor@math.okstate.edu ADDRESS: Department of Mathematics, 401 Mathematical Sciences, Oklahoma State University, Stillwater, OK 74078-1058, U.S.A. 